\title{
Characterising the interaction between the COPII component SEC24C and the human serotonin transporter
}

\author{
Florian Koban, Sonja Sucic ${ }^{*}$
}

From 17th Scientific Symposium of the Austrian Pharmacological Society (APHAR). Joint meeting with the Hungarian Society of Experimental and Clinical Pharmacology (MFT)

Innsbruck, Austria. 29-30 September 2011

\section{Background}

The serotonin transporter (SERT) belongs to the SLC6 family of neurotransmitter transporters, which mediate reuptake of previously released neurotransmitters from the synapse. Mutation of C-terminus residues RI607608 to alanine results in intracellular retention of SERT [1]. We subsequently showed that SERT depends on the COPII component SEC24C for its ER export and proposed RI607-608 as a putative interaction site on SERT for SEC24 proteins [2]. The aim of our current study is to characterise the nature of ER export of monoamine transporters.

\section{Methods}

Using siRNAs to knock down SEC24 isoforms A-D in HeLa cells, we screened a series of double and truncation mutants generated along the C-terminus of SERT. HeLa cells were transfected with Sec24 siRNAs and, after $48 \mathrm{~h}$, with YFP-tagged transporter plasmids. Functional effects of SEC24A-D knockdowns were determined by substrate uptake assays.

\section{Results}

Export of the IK $(609,610)$ AA-SERT mutant was not sensitive to knockdown of Sec24C. Remarkably, the closely related transporters for dopamine (DAT) and noradrenaline (NET), rely on Sec24D, and not C, for their ER export [2]. Accordingly, we replaced $\mathrm{K} 610$ by a tyrosine residue $(\mathrm{Y})$ to switch the SERT export motif to a NET/ DAT motif. The resulting K610Y-SERT mutant was more sensitive to the knockdown of SEC24D than of SEC24C. These observations predicted that SLC6 family members with a K-residue at the pertinent position ought to be clients of Sec24C. This prediction was verified by examining mGAT4.

\section{Conclusions}

The data imply that residue K610 and the equivalent residues in other transporters specify which SEC24 paralogue is recruited for ER export. These export signals work independently because a concatemer of SERT and GAT-1 is affected by depletion of both SEC24C and SEC24D.

\section{Acknowledgements}

This work was supported by SFB35.

Published: 5 September 2011

\section{References}

1. El-Kasaby A, Just H, Malle E, Stolt-Bergner PC, Sitte HH, Freissmuth M, Kudlacek O: Mutations in the carboxyl-terminal SEC24 binding motif of the serotonin transporter impair folding of the transporter. J Biol Chem 2010, 285:39201-39210.

2. Sucic S, El-Kasaby A, Kudlacek $O$, Sarker S, Sitte HH, Marin P, Freissmuth M: The serotonin transporter is an exclusive client of the coat protein complex II (COPII) component Sec24C. J Biol Chem 2011, 286:16482-16490.

\section{doi:10.1186/1471-2210-11-S2-A31}

Cite this article as: Koban and Sucic: Characterising the interaction between the COPII component SEC24C and the human serotonin transporter. BMC Pharmacology 2011 11(Suppl 2):A31.

\footnotetext{
* Correspondence: sonja.sucic@meduniwien.ac.at Institute of Pharmacology, Center of Physiology and Pharmacology, Medical University of Vienna, 1090 Vienna, Austria
} 